\title{
Second-Order Sliding Mode Guidance Law considering Second-Order Dynamics of Autopilot
}

\author{
Kuanqiao Zhang $(\mathbb{D}$ and Suochang Yang $\mathbb{1}$ \\ Missile Engineering Department, Army Engineering University, Shijiazhuang 050003, China \\ Correspondence should be addressed to Suochang Yang; ysuochang@163.com
}

Received 14 March 2019; Accepted 18 April 2019; Published 9 June 2019

Academic Editor: Yongji Wang

Copyright ( 2019 Kuanqiao Zhang and Suochang Yang. This is an open access article distributed under the Creative Commons Attribution License, which permits unrestricted use, distribution, and reproduction in any medium, provided the original work is properly cited.

\begin{abstract}
Aiming at the requirement that some missiles need to meet certain impact angles when attacking targets, we consider the secondorder dynamic characteristics of autopilot, thereby proposing a second-order sliding mode guidance law with impact angle constraint. Firstly, based on the terminal sliding mode control, we design a fast nonsingular terminal sliding mode guidance law with impact angle constraint. Based on the second-order sliding mode control, a second-order sliding mode guidance law with impact angle constraint is proposed. We have proved its finite time convergence characteristics and presented the specific convergence time expression. Subsequently, the dynamic characteristics of the autopilot are approximated to the second-order link. Combined with the dynamic surface control theory, we proposed a second-order sliding mode guidance law considering the second-order dynamic characteristics of the autopilot and proved its finite-time convergence characteristics. Finally, the effectiveness and superiority of the proposed guidance law are verified by comparative simulation experiments.
\end{abstract}

\section{Introduction}

In modern warfare, many types of missiles need to hit the target with certain impact angles to increase the damage efficiency of the warhead. Therefore, the impact angle constraint is a problem that needs to be considered in the guidance law design. After more than four decades of development, the research results of the guidance law with impact angle constraint are abundant. Examples are the optimal guidance laws, the improved proportional guidance laws, and the variable structure guidance laws [1]. However, the autopilot is often regarded as an ideal link in the design process of the guidance law. It is easy to ignore the influence of the autopilot dynamic characteristics on the guidance performance. Often, the dynamic characteristics of the autopilot can degrade the guidance performance, especially for attacking maneuvering targets. Therefore, the influence of the dynamic characteristics of the autopilot should also be considered in the design of guidance law.

Since the sliding mode variable structure control is invariant to the obstruction on the sliding mode, it is widely used in the design of the guiding law. In [2], the line-of-sight angular velocity and impact angle constraint have been used as the sliding surface, and the sliding mode variable structure control has been applied to design the guidance law with impact angle constraint. The adaptive exponential approach law has been used to design the sliding mode guidance law, which increases the adaptability and dynamic performance of the guidance law.

The sliding mode surfaces of the above guidance laws are all linear functions, which cannot make the system states converge to the equilibrium point in finite time, while the terminal sliding mode control adopts a nonlinear function as the sliding mode surface, which can realize the finite-time convergence of the system states [3]. Based on the terminal sliding mode control, several finite-time convergence guidance laws with impact angle constraint have been designed in [4-7], which reduce the convergence time, but the negative exponential term in the guidance command will cause singularity problem. In order to solve the singular problem, a nonsingular terminal sliding mode control method was proposed in [8]. This method uses a nonsingular terminal sliding mode function to design the sliding mode surface, which has the similar form to the terminal sliding mode 
sliding surface and finite-time convergence characteristics. Based on nonsingular terminal sliding mode control, the nonsingular terminal sliding mode surface is selected in [9], which proposed a guidance law with impact angle constraint but the convergence speed is slow. Zhou et al. [10] proposed two kinds of guidance laws, using the exponential approach law and the fast power approach law. In this method, the extended state observer is used to estimate the target motion information, which improves the guidance performance and convergence speed. In [11], a fast-convergent nonsingular terminal sliding surface is presented. This method improves the performance of the adaptive exponential approach law. In this method, an adaptive nonsingular terminal sliding mode guidance law with impact angle constraint was proposed. This scheme shows a good guidance performance.

The above methods on the guidance laws with impact angle constraints, whether based on linear sliding mode control or nonsingular terminal sliding mode control, are all based on the first-order sliding mode control theory. Although the first-order sliding mode control can meet the requirements of the finite-time convergence characteristic of the guidance law, the guidance law expression contains discontinuous switching items, which is easy to cause chattering. This is a problem inherent in first-order sliding mode control. Therefore, most of the literatures have smoothed the switching term [12] to weaken the chattering, but they also change the inherent structure of the sliding mode control and reduce the robustness. To solve this problem, a second-order sliding mode control method was proposed in $[13,14]$. This method is robust and efficient to implement while showing limited chattering. A second-order sliding mode guidance law is designed by adopting this scheme. The guidance law can achieve finite-time convergence of system states. In [15], based on the second-order sliding mode superhelical algorithm and combined with the terminal sliding surface, a second-order sliding mode guidance law with impact angle constraint is designed, and the highorder sliding mode differentiator is used to estimate the target maneuver information. In [16], a PID sliding surface is designed, and combined with a second-order sliding mode spiral algorithm, a second-order sliding mode guidance law with terminal angle constraint is presented, and its finitetime convergence property is proved based on the Lyapunov stability theory.

Due to the aerodynamic force and the delay characteristics of the missile's hardware, the missile autopilot will have dynamic delays in the atmosphere, which has a great impact on the guidance effect of the missile. If the dynamic characteristics of the autopilot are not considered, it is difficult to guarantee the guidance accuracy. In the past, the design of the guidance law considering the dynamic characteristics of the missile autopilot mostly adopted the backstepping method, but this method requires continuous derivation of the model, resulting in the high-order derivative of the states in the guidance law expression. It is difficult to measure, so this method is difficult to use in practice. In [17], the autopilot is regarded as the first-order link, and the nonlinear backstepping design method is used to design the guidance law. However, the guidance law adopts the linear sliding mode surface, which cannot guarantee the finite-time convergence of the system states. In [18], a sliding mode guidance law is modified based on sliding mode control. The first-order delay characteristic of the autopilot is considered. However, the guidance law expression contains the second derivative of the line-of-sight angle, which cannot be directly measured. In [19], the autopilot has been also approximated as a first-order link. The guidance law has been presented based on nonsingular terminal sliding mode control and dynamic surface control, and the extended state observer is used to estimate the target maneuver information. In [20], combined with terminal sliding mode control and second-order sliding mode control, the autopilot has been regarded as the firstorder link, a second-order sliding mode guidance law is proposed, and the finite-time convergence differentiator is designed to suppress the angular rate error. The guidance law has better guidance performance, but it includes the third-order derivative of the relative distance and the second derivative of the line-of-sight angle, so that the form is more complicated.

The literatures reviewed above approximate the missile autopilot to a first-order inertia link. In fact, the autopilot generally has high-order dynamic characteristics. If it is approximated as a high-order link, it can simulate the dynamic characteristics of the pilot, but the form of the guidance law is too complicated. In order to solve this problem, some authors approximate the autopilot as a second-order link, which reduces the complexity of the form of the guidance law while being close to the actual dynamic characteristics of the driver. A guidance law was proposed in [21] which considers the second-order dynamic characteristics of the missile autopilot based on dynamic surface control. In [22], based on dynamic surface control, a new guidance law considering the second-order dynamic characteristics of the autopilot was also proposed. The extended state observer is used to estimate the target motion information, but the selected sliding surface is more complicated.

Aiming at the problem of impact angle constraint, a second-order sliding mode guidance law considering the second-order dynamic characteristics of the autopilot with impact angle constraints is designed in this paper. We proved its global finite-time convergence characteristics and give the expression of convergence time. The guidance law solves the singular problem of the guidance laws in [4-7] and improves the global fast convergence of the system state variables, which solves the problem that the convergence speed is slow in [9]. The law compensates the dynamic characteristics of the autopilot effectively. Finally, the effectiveness and superiority of the guidance law are verified by comparative simulation experiments.

\section{Relative Dynamics between Missile and Target}

The relative motion relationship of the missile and target is established on the inertial coordinate system $O X Y Z$ as shown in Figure 1. $O X_{\mathrm{S}} Y_{\mathrm{S}} Z_{\mathrm{S}}$ is the line-of-sight (LOS) coordinate system. $\mathrm{M}$ and $\mathrm{T}$ represent the missile and target, respectively. $r$ denotes the relative distance of the missile and target. $q$ and 


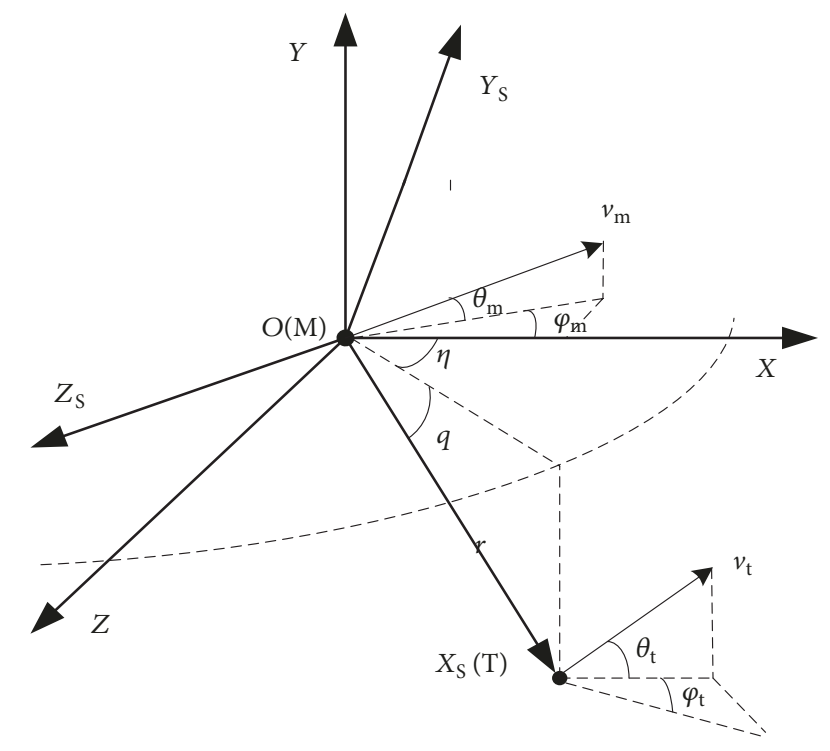

Figure 1: Three-dimensional missile-target geometry.

$\eta$ are the LOS dip angle and slip angle, respectively. $v_{\mathrm{m}}$ and $v_{\mathrm{t}}$ are the speeds of the missile and the target, respectively. $\theta_{\mathrm{m}}$ and $\varphi_{\mathrm{m}}$ represent the missile ballistic dip angle and slip angle, respectively. $\theta_{\mathrm{t}}$ and $\varphi_{\mathrm{t}}$ are the target track dip angle and slip angle.

According to the relative motion relationship of missile and target, the relative motion equations [23] can be obtained as follows:

$$
\begin{aligned}
\ddot{r}-r \dot{q}^{2}-r \dot{\eta}^{2} \cos ^{2} q & =a_{\mathrm{tr}}-a_{\mathrm{m} r} \\
r \ddot{q}+2 \dot{r} \dot{q}+r \dot{\eta}^{2} \cos q \sin q & =a_{\mathrm{t} q}-a_{\mathrm{m} q} \\
-r \ddot{\eta} \cos q-2 \dot{r} \dot{\eta} \cos q+2 \dot{r} \dot{q} \dot{\eta} \sin q & =a_{\mathrm{t} \eta}-a_{\mathrm{m} \eta}
\end{aligned}
$$

where $a_{\mathrm{tr}}, a \mathrm{t} q, a_{\mathrm{t} \eta}$, and $a_{\mathrm{m} r}, a_{\mathrm{m} q}, a_{\mathrm{m} \eta}$ are the components of the target and missile acceleration in the LOS coordinate system, respectively.

In order to facilitate the design of the guidance law, the relative motion of the missile and the target in threedimensional space can be decomposed into two twodimensional planes for researching, and the impact angle constraint problem mostly exists in the longitudinal plane. Therefore, we mainly study the guidance law in the longitudinal plane. It can be known from $[2,4-7,10,11,24]$ and other related literatures that the impact angle constraint problem can be transformed into the terminal LOS angle constraint problem. So in this paper we use the terminal LOS angle to represent the impact angle.

From the second equation of (1), the missile guidance system model with terminal LOS angle constraint can be described as follows:

$$
\begin{aligned}
& \dot{x}_{1}=x_{2} \\
& \dot{x}_{2}=a x_{2}+b u+d
\end{aligned}
$$

where

$$
\begin{aligned}
& a=a_{0}+\Delta a, \\
& b=b_{0}+\Delta b \\
& a_{0}=-\frac{2 \dot{r}}{r}, \\
& b_{0}=-\frac{1}{r} \\
& u=a_{\mathrm{m} q}=a_{\mathrm{m}} \cos \left(q-\theta_{\mathrm{m}}\right) \\
& d=-\dot{\theta}^{2} \cos q \sin q+\frac{a_{\mathrm{tq}}}{r}
\end{aligned}
$$

$\Delta a$ and $\Delta b$ are unknown bounded disturbances, state variables $x_{1}=q-q_{\mathrm{d}}, x_{2}=\dot{q}$, and $q_{\mathrm{d}}$ is the desired terminal LOS angle.

\section{Second-Order Sliding Mode Guidance Law with Terminal LOS Angle Constraint}

Before the design of the guidance law, we assume that the missile autopilot is the ideal link. That is, the guidance command has $a_{\mathrm{c}}=a_{\mathrm{m}}$. The traditional terminal sliding mode guidance law expression contains the negative exponent term, which will produce singular problem. In order to overcome the singular problem, a nonsingular terminal sliding surface has been proposed and used in the design of the guiding law. In order to improve the global convergence speed of the system states, a nonsingular fast terminal sliding surface with the terminal LOS angle constraint is designed as

$$
s=x_{1}+k_{1} x_{1}^{\alpha}+k_{2} x_{2}^{\beta}
$$

where $k_{1}, k_{2}>0, \alpha=p_{1} / p_{2}>1, \beta=p_{1} / p_{2}, 1<\beta<2, p_{1}, p_{2}, p_{3}$, $p_{4}$ are all odd.

Differentiating (4) with respect to time gives

$$
\dot{s}=x_{2}+\alpha k_{1} x_{1}^{\alpha-1} x_{2}+\beta k_{2} x_{2}^{\beta-1} \dot{x}_{2}
$$

Substituting (2) into (5), we obtain

$$
\begin{aligned}
\dot{\mathcal{s}}= & \left(1+\alpha k_{1} x_{1}^{\alpha-1}\right) x_{2}+\beta k_{2} x_{2}^{\beta-1}\left(a x_{2}+b u+d\right) \\
= & \left(1+\alpha k_{1} x_{1}^{\alpha-1}\right) x_{2}+a_{0} \beta k_{2} x_{2}^{\beta}+\beta k_{2} x_{2}^{\beta-1} b_{0} u \\
& +\beta k_{2} x_{2}^{\beta-1}\left(\Delta a x_{2}+\Delta b u+d\right)
\end{aligned}
$$

Subsequently, we design the sliding mode guidance law as

$$
\begin{aligned}
u= & \left(\beta k_{2} x_{2}^{\beta-1} b_{0}\right)^{-1}\left[-a_{0} \beta k_{2} x_{2}^{\beta}-\left(1+\alpha k_{1} x_{1}^{\alpha-1}\right) x_{2}\right] \\
& +u_{1}=-2 \dot{r} x_{2}+\frac{r}{k_{2} \beta} x_{2}^{2-\beta}\left(1+\alpha k_{1} x_{1}^{\alpha-1}\right)+u_{1}
\end{aligned}
$$

Combining (6) and (7) gives

$$
\dot{s}=\beta k_{2} x_{2}^{\beta-1}\left(\Delta a x_{2}+\Delta b u+d\right)+u_{1}
$$


Assumption 1. Suppose $\varphi(\mathbf{x}, t)=\beta k_{2} x_{2}^{\beta-1}\left(\Delta a x_{2}+\Delta b u+d\right)$ satisfies

$$
|\dot{\varphi}(\mathbf{x}, t)| \leq \varepsilon
$$

where $\varepsilon>0$. So $\varphi(\mathbf{x}, t)$ can be regarded as the total disturbance of the guidance system and is a bounded uncertainty function.

According to (8) and (9), it holds that

$$
\dot{s}=\varphi(\mathbf{x}, t)+u_{1}
$$

It can be seen from (10) that the control command $u_{1}$ is in the differential equation of the sliding surface $s$. Therefore, the second-order sliding mode super-twisting algorithm can be used to design the control instruction $u_{1}$ as follows [25]:

$$
\begin{aligned}
u_{1} & =-k_{3}|s|^{1 / 2} \operatorname{sgn}(s)+\sigma \\
\dot{\sigma} & =-k_{4} \operatorname{sgn}(s)
\end{aligned}
$$

where $\mathrm{k} 3>0, \mathrm{k} 4>0$.

Combining (7) and (11), the second-order sliding mode guidance law with terminal LOS angle constraint is obtained:

$$
\begin{aligned}
a_{\mathrm{c}} & =\frac{u}{\cos \left(q-\theta_{\mathrm{m}}\right)}=\frac{1}{\cos \left(q-\theta_{\mathrm{m}}\right)}\left[-2 \dot{r} x_{2}\right. \\
& \left.+\frac{r}{k_{2} \beta} x_{2}^{2-\beta}\left(1+\alpha k_{1} x_{1}^{\alpha-1}\right)-k_{3}|s|^{1 / 2} \operatorname{sgn}(s)+\sigma\right] \\
\dot{\sigma} & =-k_{4} \operatorname{sgn}(s)
\end{aligned}
$$

In (12), $|s|^{1 / 2} \operatorname{sgn}(s)$ and $\sigma$ are nonsmooth continuous functions, which will greatly relieve the chattering problem in sliding mode control and help improve the stability of the system. For convenience of description, the second-order sliding mode guidance law shown in (12) is simply referred to as SOSMG.

Theorem 2. If the system (2) satisfies Assumption 1, under the guidance law (12), $x_{1}$ and $x_{2}$ can converge to the origin within a finite time, that is, the LOS angle converges to the desired terminal LOS angle, and the LOS angular velocity converges to zero.

Proof. Substituting (12) into (6), we obtain

$$
\dot{s}=\varphi(\mathbf{x}, t)-k_{3}|s|^{1 / 2} \operatorname{sgn}(s)+\sigma
$$

Defining

$$
\begin{aligned}
& w_{1}=s \\
& w_{2}=\varphi(\mathbf{x}, t)+\sigma
\end{aligned}
$$

then we have

$$
\begin{aligned}
& \dot{w}_{1}=w_{2}-k_{3}\left|w_{1}\right|^{1 / 2} \operatorname{sgn}\left(w_{1}\right) \\
& \dot{w}_{2}=\dot{\varphi}(\mathbf{x}, t)-k_{4} \operatorname{sgn}\left(w_{1}\right)
\end{aligned}
$$

Define the following Lyapunov function:

$$
\begin{aligned}
V_{1} & =2 k_{4}\left|w_{1}\right|+\frac{1}{2} w_{2}^{2}+\frac{1}{2}\left(k_{3}\left|w_{1}\right|^{1 / 2} \operatorname{sgn}\left(w_{1}\right)-w_{2}\right)^{2} \\
& =\boldsymbol{\omega}^{\mathrm{T}} \mathbf{P} \boldsymbol{\omega}
\end{aligned}
$$

where

$$
\begin{aligned}
& \boldsymbol{\omega}=\left[\begin{array}{c}
\left|w_{1}\right|^{1 / 2} \\
\operatorname{sgn}\left(w_{1}\right) \\
w_{2}
\end{array}\right] \\
& \mathbf{P}=\left[\begin{array}{cc}
2 k_{4}+\frac{1}{2} k_{3}^{2} & -\frac{1}{2} k_{3} \\
-\frac{1}{2} k_{3} & 1
\end{array}\right]
\end{aligned}
$$

Since both $k_{3}$ and $k_{4}$ are constants greater than zero, it is easy to prove that $\mathbf{P}$ is a positive definite matrix and $V_{1}$ is radially unbounded, that is

$$
\lambda_{\text {min }}(\mathbf{P})\|\boldsymbol{\omega}\|^{2} \leq V_{1} \leq \lambda_{\max }(\mathbf{P})\|\boldsymbol{\omega}\|^{2}
$$

where $\lambda_{\min }(\mathbf{P})$ and $\lambda_{\max }(\mathbf{P})$ are the maximum eigenvalue and the minimum eigenvalue of $\mathbf{P}$, respectively, and $\|\cdot\|$ represents the Euclidean norm of the matrix.

Differentiating (16) with respect to time gives

$$
\begin{aligned}
\dot{V}_{1} & =\left[\left(2 k_{4}+\frac{1}{2} k_{3}^{2}\right) \operatorname{sgn}\left(w_{1}\right)-\frac{1}{2} k_{3}\left|w_{1}\right|^{-1 / 2} w_{2}\right] \dot{w}_{1} \\
& +\left[2 w_{2}-k_{3}\left|w_{1}\right|^{1 / 2} \operatorname{sgn}\left(w_{1}\right)\right] \dot{w}_{2} \\
& =\left[\left(2 k_{4}+\frac{1}{2} k_{3}^{2}\right) \operatorname{sgn}\left(w_{1}\right)-\frac{1}{2} k_{3}\left|w_{1}\right|^{-1 / 2} w_{2}\right]\left[w_{2}\right. \\
& \left.-k_{3}\left|w_{1}\right|^{1 / 2} \operatorname{sgn}\left(w_{1}\right)\right]+\left[2 w_{2}\right. \\
& \left.-k_{3}\left|w_{1}\right|^{1 / 2} \operatorname{sgn}\left(w_{1}\right)\right]\left[\dot{\varphi}(\mathbf{x}, t)-k_{4} \operatorname{sgn}\left(w_{1}\right)\right] \\
& =-\left|w_{1}\right|^{-1 / 2}\left[\left(k_{3} k_{4}+\frac{1}{2} k_{3}^{3}\right)\left|w_{1}\right|\right. \\
& \left.-k_{3}^{2}\left|w_{1}\right|^{1 / 2} \operatorname{sgn}\left(w_{1}\right) w_{2}+\frac{1}{2} k_{3} w_{2}^{2}\right]+\dot{\varphi}(\mathbf{x}, t)\left[2 w_{2}\right. \\
& \left.-k_{3}\left|w_{1}\right|^{1 / 2} \operatorname{sgn}\left(w_{1}\right)\right] \leq-\left|w_{1}\right|^{-1 / 2} \boldsymbol{\omega}^{\mathrm{T}} \mathbf{Q} \boldsymbol{\omega}+\varepsilon \mathbf{A} \boldsymbol{\omega}
\end{aligned}
$$

where

$$
\begin{aligned}
& \mathbf{Q}=\left[\begin{array}{cc}
k_{3} k_{4}+\frac{1}{2} k_{3}^{3} & -\frac{1}{2} k_{3}^{2} \\
-\frac{1}{2} k_{3}^{2} & \frac{1}{2} k_{3}
\end{array}\right] \\
& \mathbf{A}=\left[\begin{array}{ll}
-k_{3} & 2
\end{array}\right]
\end{aligned}
$$

The matrix $\mathbf{Q}$ is a positive definite matrix. 
$\left|w_{1}\right|^{-1 / 2} \geq\|\boldsymbol{\omega}\|^{-1}$ can be obtained from $\left|w_{1}\right| \leq\|\boldsymbol{\omega}\|^{2}$, then (20) can be transformed as follows:

$$
\begin{gathered}
\dot{V}_{1} \leq-\|\boldsymbol{\omega}\|^{-1} \lambda_{\min }(\mathbf{Q})\|\boldsymbol{\omega}\|^{2}+\varepsilon\|\mathbf{A}\|\|\boldsymbol{\omega}\| \\
\leq-\left(\lambda_{\min }(\mathbf{Q})-\varepsilon\|\mathbf{A}\|\right)\|\boldsymbol{\omega}\| \\
\leq-\frac{\lambda_{\min }(\mathbf{Q})-\varepsilon\|\mathbf{A}\|}{\sqrt{\lambda_{\max }(\mathbf{P})}} V_{1}^{1 / 2} \\
\text { If } \rho=\lambda_{\min }(\mathbf{Q})-\varepsilon\|\mathbf{A}\|>0 \\
\dot{V}_{1} \leq-\frac{\rho}{\sqrt{\lambda_{\max }(\mathbf{P})}} V_{1}^{1 / 2}
\end{gathered}
$$

According to the finite-time stability theory [26], $\omega$ can converge to zero in a finite time, then $s$ can converge to zero in a finite time, and the convergence time is

$$
T_{\mathrm{r}} \leq \frac{2 \sqrt{\lambda_{\max }(\mathbf{P})}}{\rho} V_{1}^{1 / 2}(0)
$$

where $V_{1}(0)$ is the initial value of $V_{1}$.

When the system state converges to the sliding surface, $s$ $=0$, which is obtained by (4)

$$
x_{1}+k_{1} x_{1}^{\alpha}+k_{2} x_{2}^{\beta}=0
$$

Define the following Lyapunov function

$$
V_{2}=x_{1}^{2}
$$

Differentiating (26) with respect to time and combining with (25) gives

$$
\begin{aligned}
\dot{V}_{2} & =2 x_{1} \dot{x}_{1}=-2 k_{2}^{-1 / \beta}\left|x_{1}\right|^{(\beta+1) / \beta}\left(1+k_{1} x_{1}^{\alpha-1}\right)^{1 / \beta} \\
& <-2 k_{2}^{-1 / \beta} V_{2}^{(\beta+1) / 2 \beta}
\end{aligned}
$$

According to the finite-time stability theory, $x_{1}$ can converge to zero in a finite time. At this time, $x_{2}$ also converges to zero in a finite time, and the convergence time is

$$
T_{\mathrm{c}}<\frac{\beta k_{2}^{1 / \beta}}{\beta-1}\left|x_{1}\left(T_{\mathrm{r}}\right)\right|^{(\beta-1) / \beta}
$$

In summary, $x_{1}$ and $x_{2}$ can converge to the origin in a finite time, and the convergence time is

$$
\begin{aligned}
T & =T_{\mathrm{r}}+T_{\mathrm{c}} \\
& <\frac{2 \sqrt{\lambda_{\max }(\mathbf{P})}}{\rho} V_{1}^{1 / 2}(0)+\frac{\beta k_{2}^{1 / \beta}}{\beta-1}\left|x_{1}\left(T_{\mathrm{r}}\right)\right|^{(\beta-1) / \beta}
\end{aligned}
$$

The proof is finished.

\section{Guide Law considering the Second-Order Dynamic Characteristics of the Autopilot}

Due to the aerodynamic force and the delay characteristics of the missile's own hardware, the autopilot might have dynamic delays in the atmosphere, which has a great impact on the guidance effect of the missile. If the dynamic characteristics of the autopilot are not considered, it is difficult to guarantee the guidance accuracy.

For the guidance system (2), the missile's autopilot can be approximated as a second-order link [27]:

$$
\frac{a_{\mathrm{m}}}{A_{\mathrm{c}}}=\frac{\omega_{n}^{2}}{s^{2}+2 \xi \omega_{\mathrm{n}} s+\omega_{\mathrm{n}}^{2}}
$$

Converting to a differential equation form gives

$$
\ddot{a}_{\mathrm{m}}=-2 \xi \omega_{\mathrm{n}} \dot{a}_{\mathrm{m}}-\omega_{\mathrm{n}}^{2} a_{\mathrm{m}}+\omega_{\mathrm{n}}^{2} A_{\mathrm{c}}
$$

Combining (2) and (31) can obtain the state equation of the guidance system considering the second-order dynamic characteristics of the autopilot

$$
\begin{aligned}
{\left[\begin{array}{c}
\dot{x}_{1} \\
\dot{x}_{2} \\
\dot{x}_{3} \\
\dot{x}_{4}
\end{array}\right]=} & {\left[\begin{array}{cccc}
0 & 1 & 0 & 0 \\
0 & a & b & 0 \\
0 & 0 & 0 & 1 \\
0 & 0 & -\omega_{\mathrm{n}}^{2} & -2 \xi \omega_{\mathrm{n}}
\end{array}\right]\left[\begin{array}{c}
x_{1} \\
x_{2} \\
x_{3} \\
x_{4}
\end{array}\right]+\left[\begin{array}{c}
0 \\
0 \\
0 \\
\omega_{\mathrm{n}}^{2}
\end{array}\right] A_{\mathrm{c}} } \\
& +\left[\begin{array}{l}
0 \\
1 \\
0 \\
0
\end{array}\right] d
\end{aligned}
$$

Theorem 3. For the guidance system (32), based on Assumption 1, the second-order sliding mode guidance law considering the second-order dynamic characteristics of the autopilot is designed based on the second-order sliding mode and dynamic surface control methods as follows:

$$
\begin{aligned}
& S_{1}=s=x_{1}+k_{1} x_{1}^{\alpha}+k_{2} x_{2}^{\beta} \\
& x_{3 \mathrm{c}}=a_{\mathrm{c}}=\frac{1}{\cos \left(q-\theta_{\mathrm{m}}\right)}\left[-2 \dot{r} x_{2}\right. \\
& \left.\quad+\frac{r}{k_{2} \beta} x_{2}^{2-\beta}\left(1+\alpha k_{1} x_{1}^{\alpha-1}\right)-k_{3}|s|^{1 / 2} \operatorname{sgn}(s)+\sigma\right] \\
& \dot{\sigma}=-k_{4} \operatorname{sgn}(s) \\
& \tau_{3} \dot{x}_{3 \mathrm{~d}}+x_{3 \mathrm{~d}}=x_{3 \mathrm{c}}, \\
& x_{3 \mathrm{~d}}(0)=x_{3 \mathrm{c}}(0) \\
& S_{2}=x_{3}-x_{3 \mathrm{~d}} \\
& x_{4 \mathrm{c}}=\dot{x}_{3 \mathrm{~d}}-k_{5} S_{2} \\
& \tau_{4} \dot{x}_{4 \mathrm{~d}}+x_{4 \mathrm{~d}}=x_{4 \mathrm{c}}, \\
& x_{4 \mathrm{~d}}(0)=x_{4 \mathrm{c}}(0) \\
& S_{3}=x_{4}-x_{4 \mathrm{~d}} \\
& \dot{S}_{3}=-k_{6} S_{3}
\end{aligned}
$$




$$
\begin{aligned}
& \dot{x}_{4}=-2 \xi \omega_{\mathrm{n}} x_{4}-\omega_{\mathrm{n}}^{2} x_{3}+\omega_{\mathrm{n}}^{2} a_{\mathrm{c}} \\
& A_{\mathrm{c}}=\frac{1}{\omega_{\mathrm{n}}^{2}}\left(-k_{6} S_{3}+2 \xi \omega_{\mathrm{n}} x_{4}+\omega_{\mathrm{n}}^{2} x_{3}+\dot{x}_{4 \mathrm{~d}}\right)
\end{aligned}
$$

where $x_{3 \mathrm{c}}$ and $x_{4 \mathrm{c}}$ are virtual control instructions. The system states $x_{1}$ and $x_{2}$ are able to converge to zero for a finite time. This means that the LOS angle converges to the desired value and the angular velocity converges to zero.

Proof. Define

$$
\begin{aligned}
& y_{3}=x_{3 \mathrm{~d}}-x_{3 \mathrm{c}} \\
& y_{4}=x_{4 \mathrm{~d}}-x_{4 \mathrm{c}}
\end{aligned}
$$

Differentiating (34) with respect to time

$$
\begin{aligned}
& \dot{y}_{3}=-\frac{y_{3}}{\tau_{3}}-\dot{x}_{3 \mathrm{c}} \\
& \dot{y}_{4}=-\frac{y_{4}}{\tau_{4}}-\dot{x}_{4 \mathrm{c}}
\end{aligned}
$$

Available from (33)

$$
\begin{aligned}
\dot{S}_{1} & =\beta k_{2} x_{2}^{\beta-1} b_{0}\left(x_{3}-x_{3 c}\right)+\varphi(x, t)+u_{1} \\
& =\beta k_{2} x_{2}^{\beta-1} b_{0}\left(S_{2}+y_{3}\right)+\varphi(x, t)+u_{1} \\
\dot{S}_{2} & =S_{3}+y_{4}-k_{5} S_{2}
\end{aligned}
$$

Define the following Lyapunov function

$$
V_{3}=\frac{S_{1}^{2}+S_{2}^{2}+S_{3}^{2}+y_{3}^{2}+y_{4}^{2}}{2}
$$

Differentiating (37) with respect to time gives

$$
\begin{aligned}
\dot{V}_{3}= & S_{1} \dot{S}_{1}+S_{2} \dot{S}_{2}+S_{3} \dot{S}_{3}+y_{3} \dot{y}_{3}+y_{4} \dot{y}_{4} \\
= & \beta k_{2} x_{2}^{\beta-1} b_{0} S_{1}\left(S_{2}+y_{3}\right)+S_{1}\left(\varphi(x, t)+u_{1}\right) \\
& +S_{2}\left(S_{3}+y_{4}-k_{5} S_{2}\right)-k_{6} S_{3}^{2}-\frac{y_{3}^{2}}{\tau_{3}}-y_{3} \dot{x}_{3 c} \\
& -\frac{y_{4}^{2}}{\tau_{4}}-y_{4} \dot{x}_{4 c}
\end{aligned}
$$

It can be seen from the proof of Theorem 2, if $\lambda_{\min }(\mathbf{Q})-$ $\varepsilon\|\mathbf{A}\|>0, \boldsymbol{\omega}$ can converge to zero in a finite time, then $\varphi(x, t)$ $+u_{1}$ can converge to zero in a finite time. Therefore, (38) can be converted into a new form:

$$
\begin{aligned}
\dot{V}_{3}= & \beta k_{2} x_{2}^{\beta-1} b_{0} S_{1}\left(S_{2}+y_{3}\right)+S_{2}\left(S_{3}+y_{4}-k_{5} S_{2}\right) \\
& -k_{6} S_{3}^{2}-\frac{y_{3}^{2}}{\tau_{3}}-y_{3} \dot{x}_{3 \mathrm{c}}-\frac{y_{4}^{2}}{\tau_{4}}-y_{4} \dot{x}_{4 \mathrm{c}}
\end{aligned}
$$

It is known from [28] that there are positive real numbers $M_{1}>0$ and $M_{2}>0$, so that $\left|\dot{x}_{3 \mathrm{c}}\right|<M_{1},\left|\dot{x}_{4 \mathrm{c}}\right|<M_{2}$. Since $b_{0}<0$, (39) can be scaled as

$$
\begin{aligned}
\dot{V}_{3} \leq & \beta k_{2} x_{2}^{\beta-1} b_{0}\left(S_{1} S_{2}+S_{1} y_{3}\right)+S_{2} S_{3}+S_{2} y_{4}-k_{5} S_{2}^{2} \\
& -k_{6} S_{3}^{2}-\frac{y_{3}^{2}}{\tau_{3}}+M_{1}\left|y_{3}\right|-\frac{y_{4}^{2}}{\tau_{4}}+M_{2}\left|y_{4}\right| \\
\leq & \beta k_{2} x_{2}^{\beta-1} b_{0}\left[\frac{S_{1}^{2}+S_{2}^{2}}{2}+\frac{S_{1}^{2}+y_{3}^{2}}{2}\right]+\frac{S_{2}^{2}+S_{3}^{2}}{2} \\
& -k_{5} S_{2}^{2}+\frac{S_{2}^{2}+y_{4}^{2}}{2}-k_{6} S_{3}^{2}-\frac{y_{3}^{2}}{\tau_{3}}-\frac{y_{4}^{2}}{\tau_{4}}+M_{1}^{2} y_{3}^{2} \\
& +M_{2}^{2} y_{4}^{2}+\frac{1}{2} \\
\leq & \beta k_{2} x_{2}^{\beta-1} b_{0} S_{1}^{2}-\left(k_{5}-\frac{\beta k_{2} x_{2}^{\beta-1} b_{0}}{2}-1\right) S_{2}^{2} \\
& -\left(k_{6}-\frac{1}{2}\right) S_{3}^{2}-\left(\frac{1}{\tau_{3}}-\frac{\beta k_{2} x_{2}^{\beta-1} b_{0}}{2}-M_{1}^{2}\right) y_{3}^{2} \\
& -\left(\frac{1}{\tau_{4}}-\frac{1}{2}-M_{1}^{2}\right) y_{4}^{2}+\frac{1}{2}
\end{aligned}
$$

Selecting the following parameters

$$
\begin{aligned}
& k_{5}>1+\frac{\beta k_{2} x_{2}^{\beta-1} b_{0}}{2} \\
& k_{6}>\frac{1}{2} \\
& \frac{1}{\tau_{3}}>M_{1}^{2}+\frac{\beta k_{2} x_{2}^{\beta-1} b_{0}}{2} \\
& \frac{1}{\tau_{4}}>\frac{1}{2}+M_{1}^{2}
\end{aligned}
$$

one can obtain

$$
\dot{V}_{3} \leq-2 \mu V_{3}+\frac{1}{2}
$$

where

$$
\begin{array}{r}
\mu=\min \left\{-\beta k_{2} x_{2}^{\beta-1} b_{0}, k_{5}+\frac{\beta k_{2} x_{2}^{\beta-1} b_{0}}{2}-1, k_{6}\right. \\
\left.-\frac{1}{2}, \frac{1}{\tau_{3}}+\frac{\beta k_{2} x_{2}^{\beta-1} b_{0}}{2}-M_{1}^{2}, \frac{1}{\tau_{4}}-\frac{1}{2}-M_{1}^{2}\right\}
\end{array}
$$

Solving (42) yields

$$
0 \leq V_{3}(t) \leq \frac{1}{4 \mu}+\left(V_{3}(0)-\frac{1}{4 \mu}\right) e^{-2 \mu t}
$$

It can be seen from (44) that $V_{3}(t)$ is bounded; therefore, $S_{1}, S_{2}, S_{3}, y_{3}$, and $y_{4}$ are bounded. If the parameters are chosen 
properly, they can be arbitrarily small, which can ensure that $V_{3}(t)$ converges to zero in a finite time, which ensures the stability of the system, and $S_{1}$ can also converge to zero in a limited time. When $S_{1}=0$, by Theorem 2 , it is easy to prove that the system states $x_{1}$ and $x_{2}$ can converge to zero in a finite time. The proof is finished.

To simplify the guidance law (33), the second-order sliding mode guidance law with terminal LOS angle constraint considering the second-order dynamic characteristics of the autopilot is considered as

$$
\begin{aligned}
A_{\mathrm{c}} & =\frac{1}{\omega_{\mathrm{n}}^{2}}\left[\left(2 \xi \omega_{\mathrm{n}}-k_{6}\right) x_{4}+\left(\omega_{\mathrm{n}}^{2}-\frac{k_{5}}{\tau_{4}}\right) x_{3}\right. \\
& \left.+\frac{\tau_{3} k_{5}-1}{\tau_{3} \tau_{4}} x_{3 \mathrm{~d}}+\frac{\tau_{4} k_{6}-1}{\tau_{4}} x_{4 \mathrm{~d}}+\frac{a_{\mathrm{c}}}{\tau_{3} \tau_{4}}\right]
\end{aligned}
$$

Remark 4. Note that $x_{3 \mathrm{~d}}$ and $x_{4 \mathrm{~d}}$ in the guidance law (45) need to be solved according to (33), which is more complicated. Comparing the range of values of $k_{5}$ and $1 / \tau_{3}, k_{6}$ and $1 / \tau_{4}$, we learn that the range of values is basically coincident, so we can make $k_{5}=1 / \tau_{3}, k_{6}=1 / \tau_{6}$. At this time, the $x_{3 \mathrm{~d}}$ and $x_{4 \mathrm{~d}}$ in the guidance law (45) can be eliminated, and the number of parameters in the guidance law can be reduced without affecting the overall performance. The simplified form of the guidance law is described as

$$
A_{\mathrm{c}}=\frac{1}{\omega_{\mathrm{n}}^{2}}\left[\left(2 \xi \omega_{\mathrm{n}}-k_{6}\right) x_{4}+\left(\omega_{\mathrm{n}}^{2}-k_{5} k_{6}\right) x_{3}+k_{5} k_{6} a_{\mathrm{c}}\right]
$$

Remark 5. It can be seen from the expression of the guidance law (12) that if $q-\theta_{\mathrm{m}}=\pi / 2, a_{\mathrm{c}}$ tends to infinity, and the available overload of the missile is limited, so it is necessary to perform limiting $a_{\mathrm{c}}$ as follows:

$$
a_{\mathrm{c}}= \begin{cases}a_{\mathrm{m} \max } \operatorname{sgn}\left(a_{\mathrm{c}}\right), & \left|a_{\mathrm{c}}\right| \geq N_{\max } \cdot g \\ a_{\mathrm{c}}, & \left|a_{\mathrm{c}}\right|<N_{\max } \cdot g\end{cases}
$$

where $N_{\max }$ is the maximum available overload of the missile and $g$ is the acceleration of gravity.

For the convenience of description, the sliding mode guidance law considering second-order dynamics of autopilot represented by (46) is simply referred to as SODSMG.

\section{Simulation Analysis}

In order to evaluate the performance of the designed guidance law, this section performs simulation analysis based on ballistic simulation in different scenarios. The initial positions of the missile and the target are set as $(0 \mathrm{~m}, 0 \mathrm{~m})$ and $(10000 \mathrm{~m}, 5000 \mathrm{~m})$, respectively. The speed of the missile is $v_{\mathrm{m}}=500 \mathrm{~m} / \mathrm{s}$, and the initial dip angle is $\theta_{\mathrm{m}}=45 \mathrm{deg}$. The speed of motion is $v_{\mathrm{t}}=250 \mathrm{~m} / \mathrm{s}$ and the initial dip angle is $\theta_{\mathrm{t}}=120 \mathrm{deg}$. The maximum available overload of the missile is $20 \mathrm{~g}$. The dynamic parameters of the missile autopilot are $\xi=0.8, \omega_{\mathrm{n}}=8 \mathrm{rad} / \mathrm{s}$. The simulation step size is $0.01 \mathrm{~s}$, and the fourth-order Runge-Kutta method is used to solve the relative motion equation of the missile and target. In order to verify the superiority of the designed guidance law, this section also carries out the adaptive sliding mode guidance law (ASMG) proposed in [24] and the nonsingular terminal sliding mode guidance law (NTSMG) proposed in [10] to perform a comparative simulation. The mathematical expression of ASMG is

$$
\begin{aligned}
a_{\mathrm{c}} & =\frac{1}{n_{1} \cos \left(q-\theta_{\mathrm{m}}\right)}\left[\left(n_{2}+n_{1} n_{3}+2 n_{1}\right) v_{\mathrm{m}} x_{2}\right. \\
& \left.+\varepsilon_{1} \operatorname{sgn}\left(s_{1}\right)+\frac{n_{2} v_{\mathrm{m}}^{2}\left(n_{3}+1\right) x_{1}}{r}\right]
\end{aligned}
$$

where $s_{1}=n_{1} x_{2}+n_{2} v_{\mathrm{m}} x_{1} / r$. The mathematical expression of NTSMG is as follows:

$$
a_{\mathrm{c}}=\frac{1}{\cos \left(q-\theta_{\mathrm{m}}\right)}\left[-2 \dot{r} x_{2}+\frac{r}{k \beta} x_{2}^{2-\beta}+\varepsilon_{2} \operatorname{sgn}\left(s_{2}\right)\right]
$$

where $s_{2}=x_{1}+k x_{2}^{\beta}$.

The simulation is carried out according to three motion modes of the target, including uniform motion, circular maneuver, and sinusoidal maneuver, and $a_{\mathrm{t}}=20 \mathrm{~m} / \mathrm{s}$ for circular maneuvering, $a_{\mathrm{t}}=20 \sin (\pi \mathrm{t} / 5) \mathrm{m} / \mathrm{s}$ for sinusoidal maneuvering. The simulation results are shown in Figures $2-5$ and Table 1. Figure 2 is the relative movement trajectory curve of missile and target, Figure 3 is the curve of the LOS angle, Figure 4 is the curve of the LOS angular rate, Figure 5 is the curve of the missile overload, and Table 1 shows the simulation results of attack time, miss distance, and terminal LOS angle error under the different guiding laws, where the LOS angle errors of ASMG, NTSMG, and SOSMG are the value before divergence.

It can be seen from Figure 2 that under SOSMG and SODSMG, the trajectories of the missiles are basically coincident and smooth, and the trajectory is relatively straight at the end. This is because SOSMG and SODSMG adopt nonsingular fast terminal sliding surface. In this way, the LOS angular rate can quickly converge to near zero in a limited time. In the case of ASMG, the curvatures of the missile's motion trajectories are larger overall. For NTSMG, the trajectories of the missiles have a sharp change in curvature at the end of the guidance phase.

It can be seen from Figure 3 that under ASMG and NTSMG, the LOS angle can converge to the desired terminal LOS angles of $90 \pm 1.6 \mathrm{deg}$ and $90 \pm 2.3 \mathrm{deg}$, respectively, but there is chattering in both ends. The chattering and divergence phenomenon is mainly caused by the inherent chattering problem of the sliding mode guidance law and the dynamic characteristics of the autopilot. The chattering phenomenon of NTSMG is more obvious. This is because the ASMG adopts an adaptive exponential approach law which can weaken chattering to some extent. Under SOSMG, the LOS angle can converge to a range of $90 \pm 0.5 \mathrm{deg}$, but there is also some chattering and divergence in the final stage. The main reason is the dynamic characteristics of the autopilot. When SODSMG is used, the second-order 

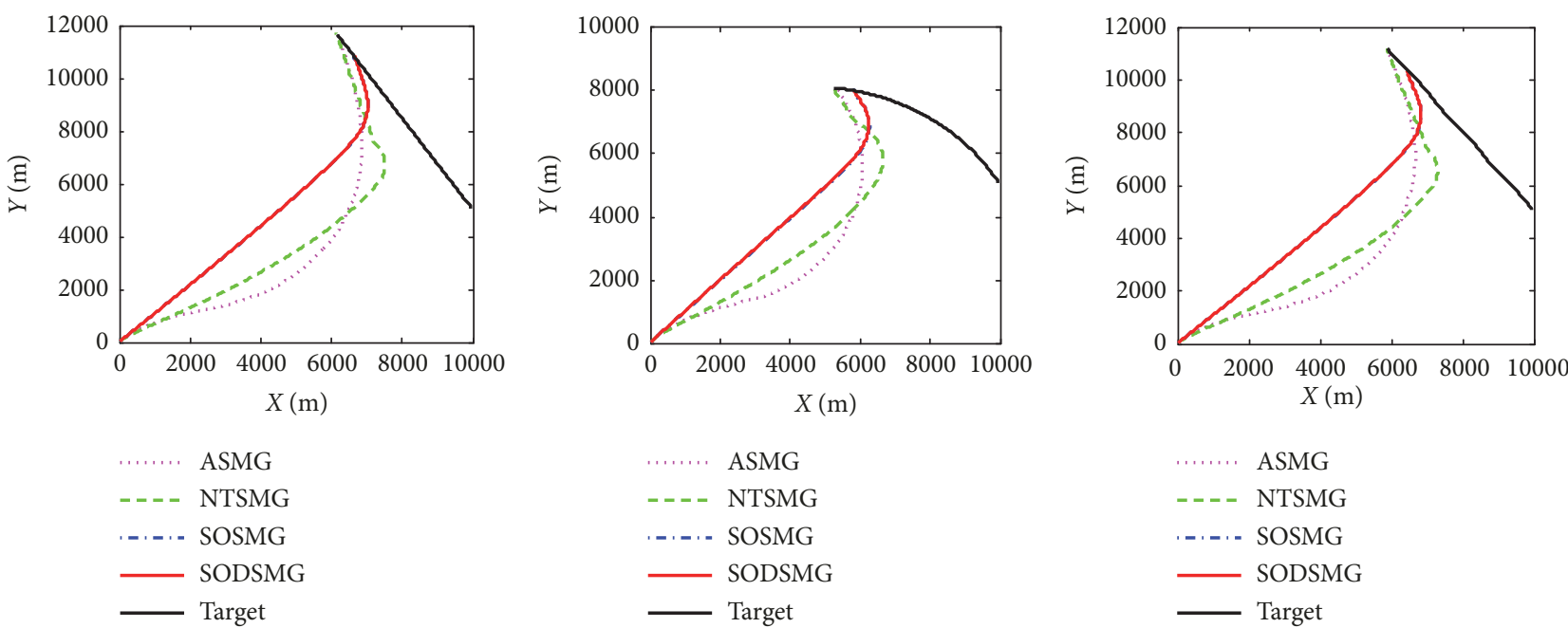

(a) Target uniform motion

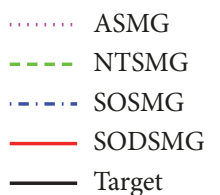

(b) Target circular maneuver

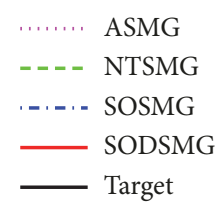

(c) Target sinusoidal maneuver

FIGURE 2: The trajectories of missile and target.

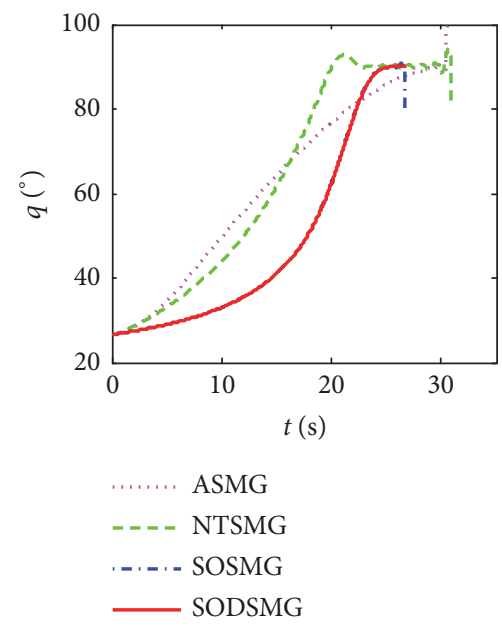

(a) Target uniform motion
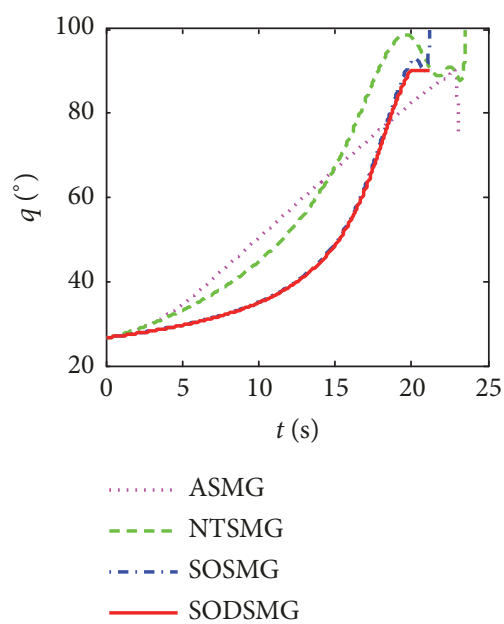

(b) Target circular maneuver
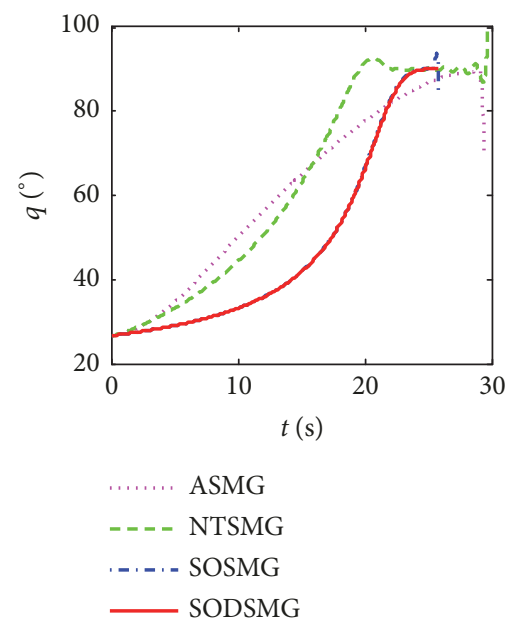

(c) Target sinusoidal maneuver

FIGURE 3: LOS angle.

TABLE 1: Simulation results of attack time, miss distance, and terminal LOS angle error.

\begin{tabular}{lcccc}
\hline Target movement & Guidance law & Attack time $(\mathrm{s})$ & Miss distance $(\mathrm{m})$ & terminal LOS angle error (deg) \\
\hline \multirow{4}{*}{ uniform motion } & ASMG & 30.51 & 5.98 & 1.6 \\
& NTSMG & 30.91 & 6.65 & 2.3 \\
& SOSMG & 26.76 & 1.66 & 0.5 \\
& SODSMG & 26.75 & 0.22 & 0.05 \\
circular maneuver & ASMG & 23.05 & 4.19 & 2.1 \\
& NTSMG & 23.58 & 7.32 & 5.6 \\
& SOSMG & 21.23 & 2.36 & 1.5 \\
SODSMG & 21.21 & 0.73 & 0.08 \\
sinusoidal maneuver & ASMG & 29.40 & 4.72 & 1.5 \\
& NTSMG & 29.79 & 7.54 & 3.2 \\
& SOSMG & 25.82 & 1.94 & 0.8 \\
& SODSMG & 25.81 & 0.46 & 0.07 \\
\hline
\end{tabular}




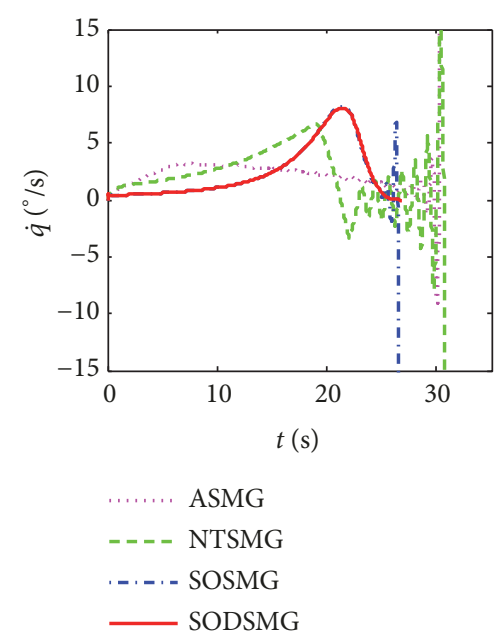

(a) Target uniform motion

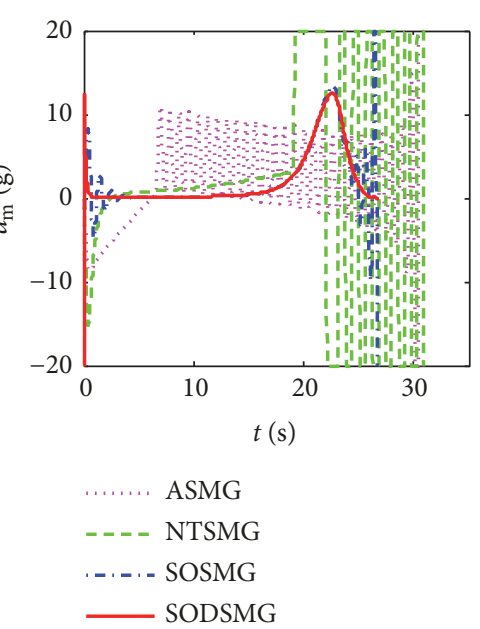

(a) Target uniform motion

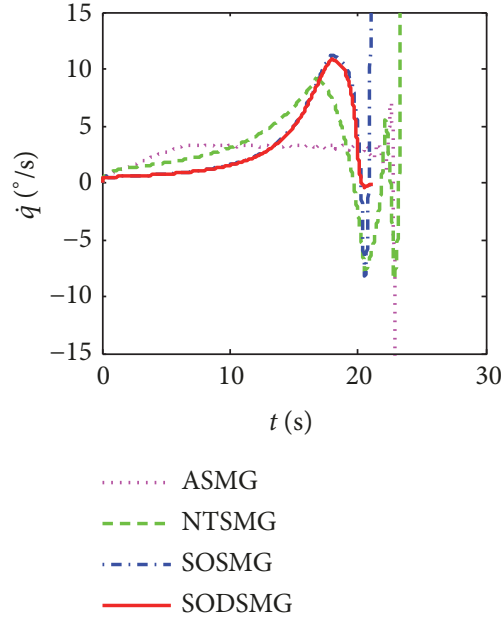

(b) Target circular maneuver

FIGURE 4: LOS angular rate.

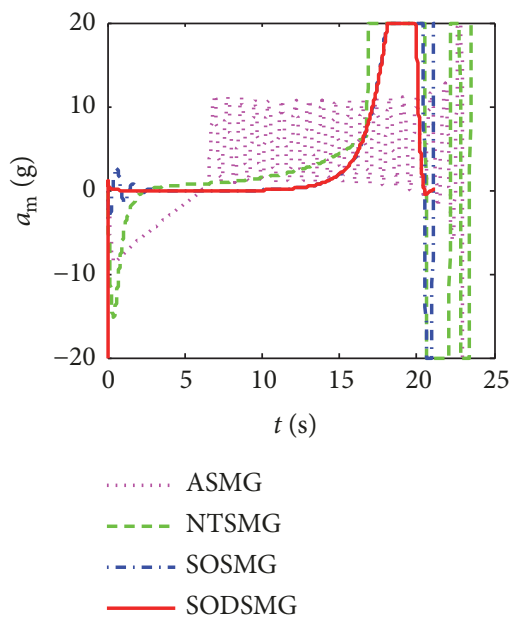

(b) Target circular maneuver

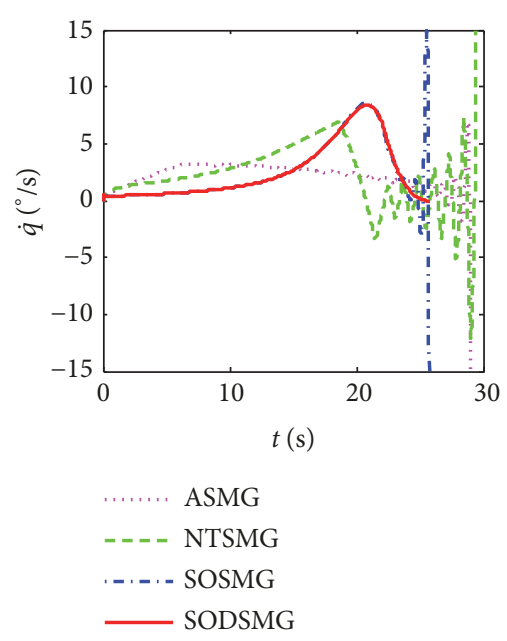

(c) Target sinusoidal maneuver

FIGURE 5: Missile overload.

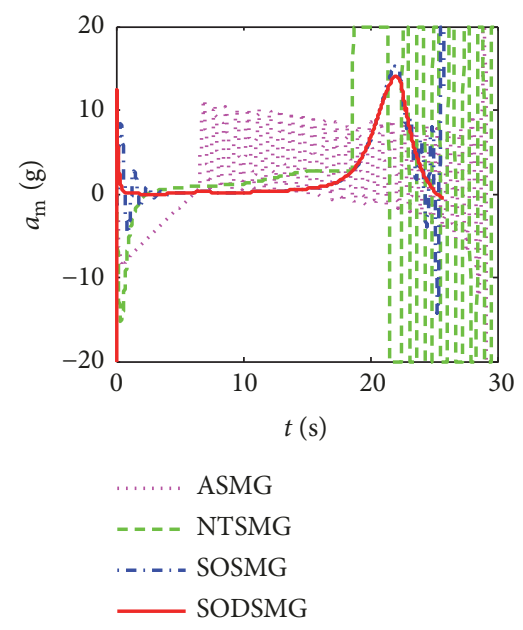

(c) Target sinusoidal maneuver sliding mode suppresses the chattering phenomenon and the dynamic surface control compensates the autopilot dynamic characteristics. The LOS angle can be strictly converged to the desired terminal LOS angle, and the chattering phenomenon is greatly weakened.

It can be seen from Figures 4 and 5 that in scenarios of ASMG and NTSMG, both the LOS angular velocity and the missile overload cannot converge to zero, and there is a large chattering phenomenon, which has diverging tendency at the end. The miss distance of the missile will be increased (as can be seen in Table 1). In the case of SOSMG, the LOS angular velocity and the overload of the missile cannot effectively converge to near zero, and there is a certain chattering phenomenon and divergence trend, but the chattering amplitude and frequency are smaller than ASMG and NTSMG. For SODSMG, both the LOS angular rate and the missile overload can effectively converge to near zero, and the chattering phenomenon is greatly reduced. It can also be seen from
Figure 5 that the missile overloads of SOSMG and SODSMG are larger in the middle of the guidance and gradually converge towards zero at the end. From the perspective of energy distribution, in the guidance middle phase, missile is flight stable and can provide a large overload so that the LOS angle and angular rate converge quickly and finitely. At the end of the guidance phase, the overload is small and there is enough overload margin to deal with the interference, so that the guidance accuracy of the missile is improved. Therefore, the performances of SOSMG and SODSMG are both significantly better than ASMG and NTSMG in terms of energy allocation.

It can be seen from Table 1 that the attack time, miss distance, and LOS angle error of SOSMG and SODSMG are significantly smaller than ASMG and NTSMG, so SOSMG and SODSMG can hit targets with shorter time and higher precision, which is of great significance on the battlefield. Due to the compensation of the autopilot's dynamic 
characteristics, the SODSMG's miss distance is smaller than SOSMG and has better guidance performance.

In summary, the guidance law SODSMG proposed in this paper compensates the dynamic characteristics of the autopilot and uses second-order sliding mode control to attenuate the buffeting. The attack time and miss distance are smaller than ASMG and NTSMG, and the LOS angle and angular rate can effectively converge to the expected value within a limited time, which verifies the effectiveness and superiority of SODSMG.

\section{Conclusion}

Aiming at guidance precision and terminal LOS angle constraint problems for some missiles, based on terminal sliding mode control, second-order sliding mode control, and dynamic surface control theories, we propose a secondorder sliding mode guidance law considering the secondorder dynamic characteristics of autopilot. We have proved its stability and finite-time convergence characteristics. The simulation results show that the proposed guidance law can make the LOS angle and angular rate converge quickly in finite time and requires fewer overloads at the end of guidance phase, which can hit the target with shorter time and higher precision.

\section{Data Availability}

The data used to support the findings of this study are included within the article.

\section{Conflicts of Interest}

The authors declare that there is no conflict of interest regarding the publication of this paper.

\section{References}

[1] H. Cai, Z.-D. Hu, and Y. Cao, "A survey of guidance law with terminal impact angle constraints," Journal of Astronautics, vol. 31, no. 2, pp. 315-323, 2010.

[2] J.-M. Song and T.-Q. Zhang, "Passive homing missile's variable structure proportional navigation with terminal angular constraint," Chinese Journal of Aeronautics, vol. 14, no. 2, pp. 83-87, 2001.

[3] C. X. Mu, X. H. Yu, and C. Y. Sun, "Phase trajectory and transient analysis for nonsingular terminal sliding mode control systems," Acta Automatica Sinica, vol. 39, no. 6, pp. 902-908, 2013.

[4] S. R. Kumar, S. Rao, and D. Ghose, "Sliding-mode guidance and control for all-aspect interceptors with terminal angle constraints," Journal of Guidance, Control, and Dynamics, vol. 35, no. 4, pp. 1230-1246, 2012.

[5] Z. Hui-bo, S. Shen-mini, X. Ming-yue, and S. Jun-hongi, "Design of terminal sliding-mode guidance law with attack angle constraints," in Proceedings of the 2013 25th Chinese Control and Decision Conference (CCDC), pp. 556-560, Guiyang, China, May 2013.
[6] Y. Zhang, M. Sun, and Z. Chen, "Finite-time convergent guidance law with impact angle constraint based on slidingmode control," Nonlinear Dynamics, vol. 70, no. 1, pp. 619-625, 2012.

[7] J. Song, S. Song, Y. Guo, and H. Zhou, "Nonlinear disturbance observer-based fast terminal sliding mode guidance law with impact angle constraints," International Journal of Innovative Computing, Information and Control, vol. 11, no. 3, pp. 787-802, 2015.

[8] Y. Feng, X. Yu, and Z. Man, "Non-singular terminal sliding mode control of rigid manipulators," Automatica, vol. 38, no. 12, pp. 2159-2167, 2002.

[9] S. R. Kumar, S. Rao, and D. Ghose, "Nonsingular terminal sliding mode guidance with impact angle constraints," Journal of Guidance, Control, and Dynamics, vol. 37, no. 4, pp. 1114-1130, 2014.

[10] H.-B. Zhou, S.-M. Song, and H.-K. Liu, "Nonsingular terminal sliding mode guidance law with impact angle constraint," Journal of Chinese Inertial Technology, vol. 22, no. 5, pp. 606618, 2014.

[11] S. Yang, K. Zhang, and P. Chen, "Adaptive terminal sliding mode guidance law with impact angle constraint," Journal of Beijing University of Aeronautics and Astronautics, vol. 42, no. 8, pp. 1566-1574, 2016.

[12] X. Wang and J. Wang, "Partial integrated guidance and control with impact angle constraints," Journal of Guidance, Control, and Dynamics, vol. 38, no. 5, pp. 925-936, 2015.

[13] A. Levant, "Principles of 2-sliding mode design," Automatica, vol. 43, no. 4, pp. 576-586, 2007.

[14] Y. B. Shtessel, I. A. Shkolnikov, and A. Levant, "Smooth secondorder sliding modes: missile guidance application," Automatica, vol. 43, no. 8, pp. 1470-1476, 2007.

[15] S. He, D. Lin, and J. Wang, "Continuous second-order sliding mode based impact angle guidance law," Aerospace Science and Technology, vol. 41, pp. 199-208, 2015.

[16] J. Guo G, T. Han, J. Zhou et al., "Second-order sliding mode guidance law with impact angle constraint," Acta Aeronautica et Astronautica Sinica, vol. 38, no. 2, article no. 320208, 2017.

[17] D. Zhou, P. P. Qu, and S. Sun, "A guidance law with terminal impact angle constraint accounting for missile autopilot," Journal of Dynamic Systems, Measurement, and Control, vol. 135, no. 5, pp. 1-8, 2013.

[18] S. Sun, H.-M. Zhang, C.-M. Yu, and Y. Meng, "Sliding-model guidance law design considering missile dynamic delay," Systems Engineering and Electronics, vol. 36, no. 8, pp. 1614-1618, 2014.

[19] S. F. Xiong, W. H. Wang, X. D. Liu et al., "Impact angle guidance law considering missile's dynamics of autopilot," Control and Decision, vol. 30, no. 4, pp. 585-592, 2015.

[20] J. Li, T. Zhang, H. M. Lei et al., "Nonsingular fast terminal second-order sliding mode guidance law with finite-time convergence," Systems Engineering and Electronics, vol. 40, no. 4, pp. 860-867, 2018.

[21] P.-P. Qu and D. Zhou, "Guidance law incorporating secondorder dynamics of missile autopilots," Systems Engineering and Electronics, vol. 33, no. 10, pp. 2263-2267, 2011.

[22] K. Zhang, S. Yang, K. Zhang, Y. Zhang, and P. Chen, "Novel guidance law accounting for dynamics of missile autopilot," Journal of Beijing University of Aeronautics and Astronautics, vol. 43, no. 8, pp. 1693-1704, 2017. 
[23] M. Golestani, I. Mohammadzaman, and A. R. Vali, "Finitetime convergent guidance law based on integral backstepping control," Aerospace Science and Technology, vol. 39, pp. 370-376, 2014.

[24] P. Wu and M. Yang, "Variable structure guidance law with terminal attack angle constraint," Journal of Solid Rocket Technology, vol. 31, no. 2, pp. 116-120, 2008.

[25] A. Levant, "Sliding order and sliding accuracy in sliding mode control," International Journal of Control, vol. 58, no. 6, pp. 12471263, 1993.

[26] Y. Hong, "Finite-time stabilization and stabilizability of a class of controllable systems," Systems \& Control Letters, vol. 46, no. 4, pp. 231-236, 2002.

[27] P. Zarchan, Tactical and strategic missile guidance, America Institute of Aeronautics and Astronautics, Reston, Va, USA, 6th edition, 2012.

[28] D. Swaroop, J. K. Hedrick, P. P. Yip, and J. C. Gerdes, "Dynamic surface control for a class of nonlinear systems," IEEE Transactions on Automatic Control, vol. 45, no. 10, pp. 1893-1899, 2000. 


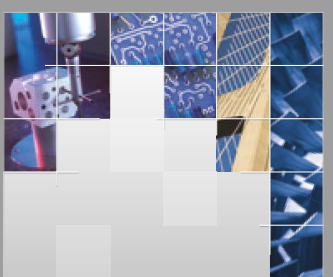

\section{Enfincering}
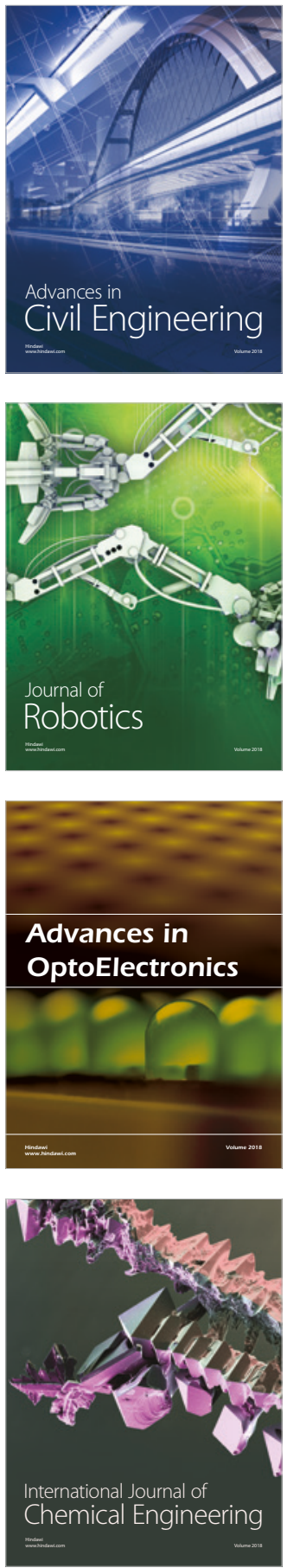

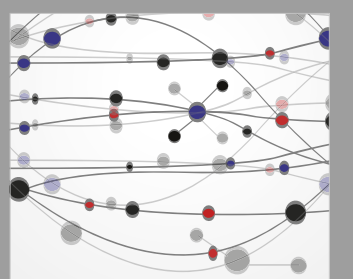

\section{Rotating \\ Machinery}

The Scientific World Journal

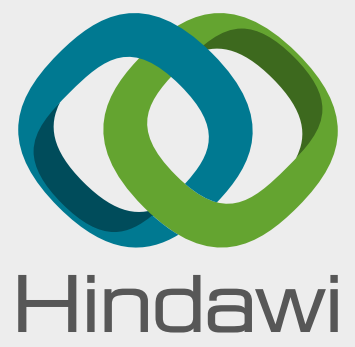

Submit your manuscripts at

www.hindawi.com
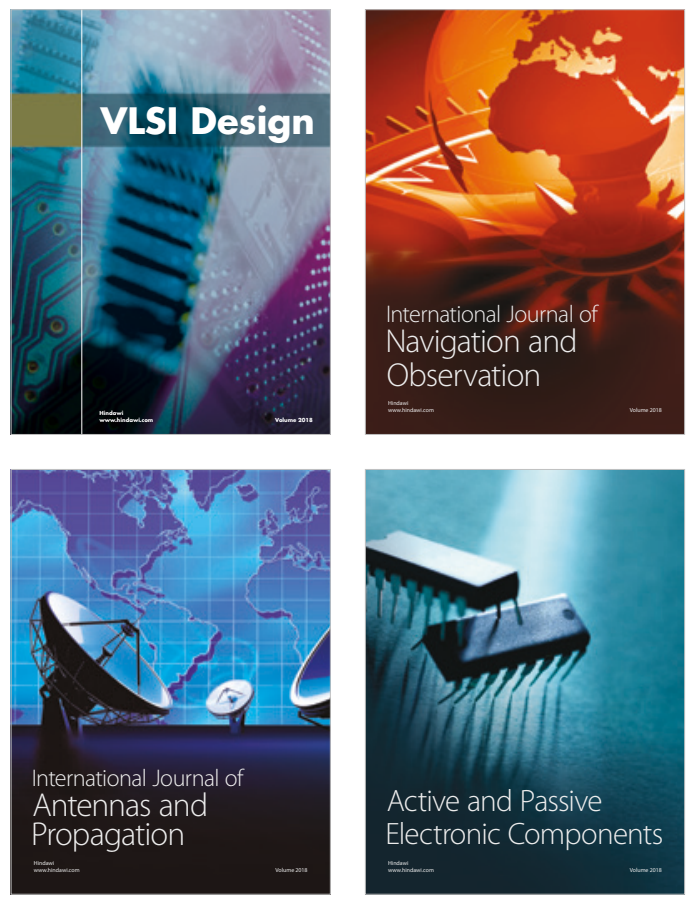
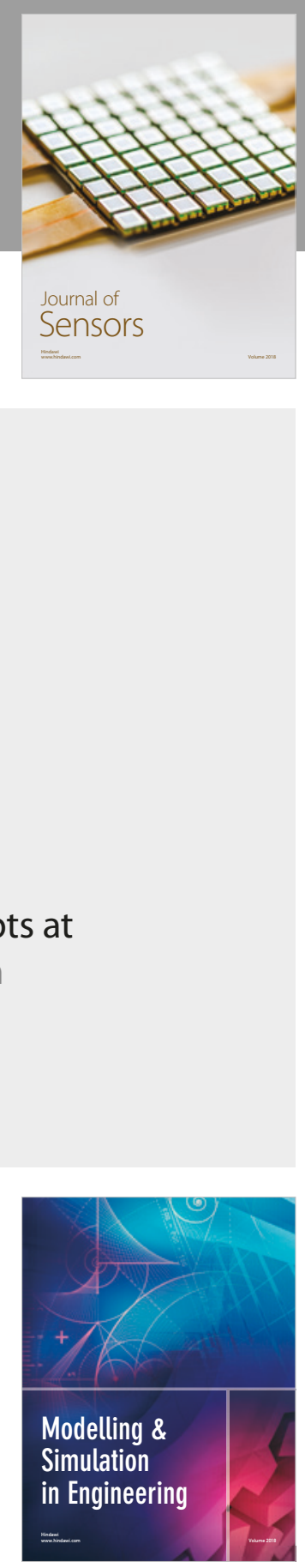

\section{Advances \\ Multimedia}
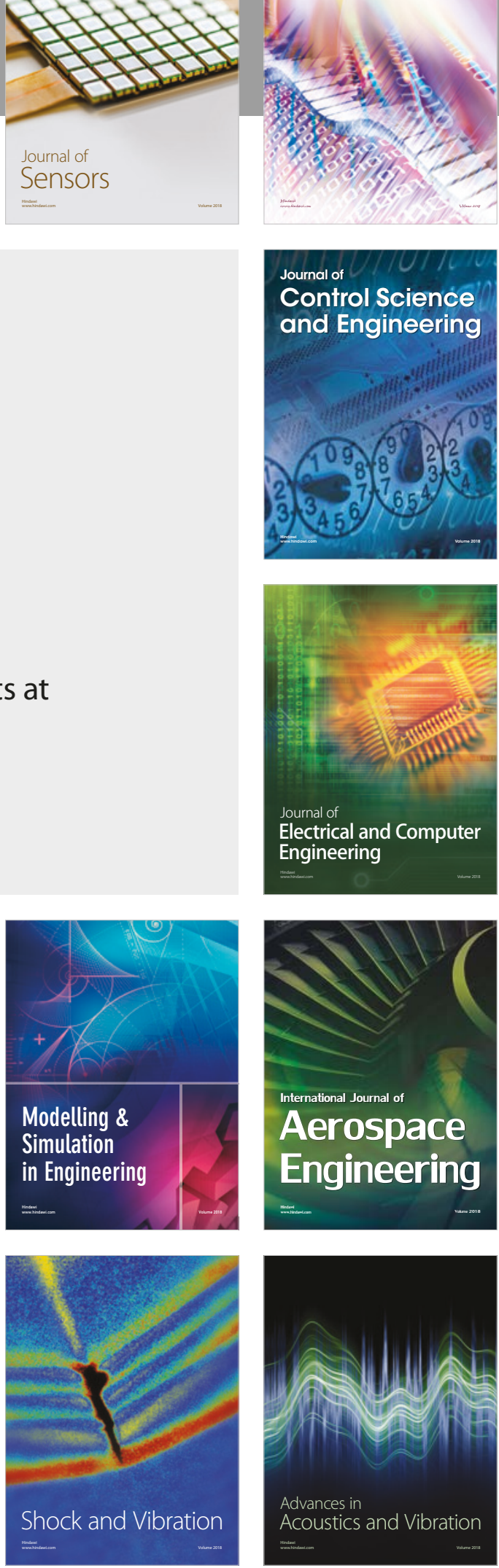